\title{
CMAJ
}

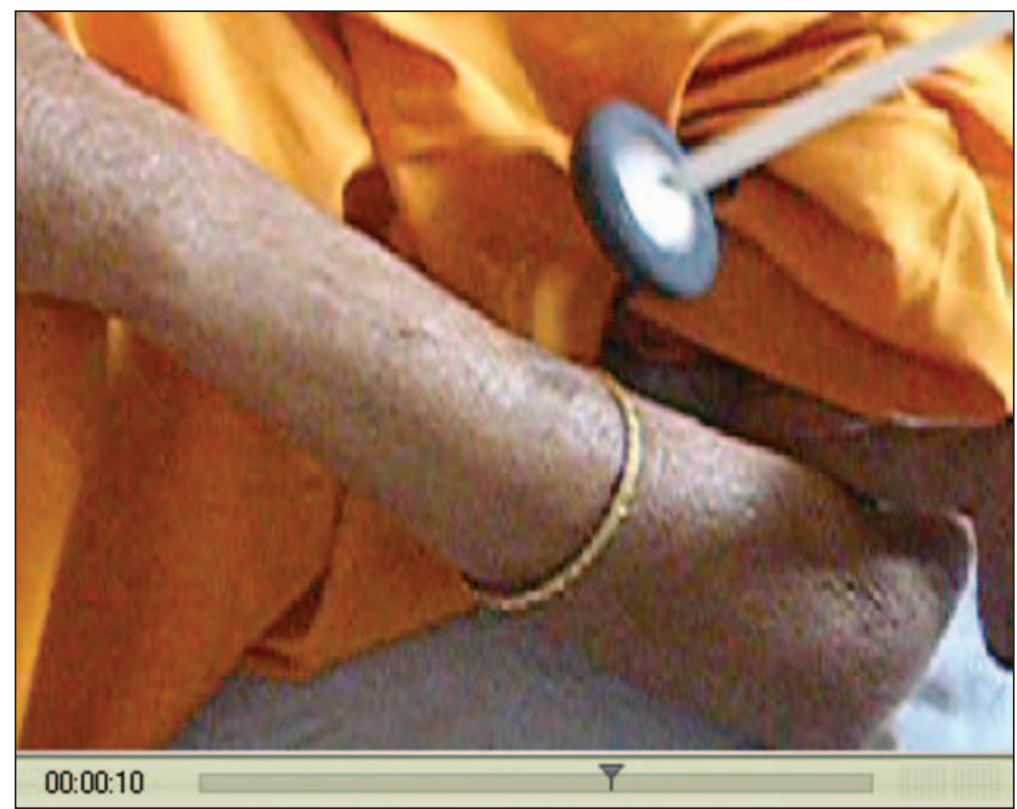

Figure 1: Screen capture from a video showing the Woltman sign in the bicep tendon of a 55-year-old woman.

\section{A classic sign of hypothyroidism: a video demonstration}

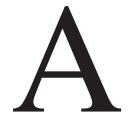

55-year-old woman presented to hospital with a 2-month history of facial puffiness, constipation, hoarse voice, fatigue and cold intolerance. She had no history of illness, and she was not taking any medication. On examination, her vital signs were normal, and she was not in distress. Her voice was hoarse, and she had facial and pedal edema, yellow skin and delayed relaxation of deep tendon reflexes in her upper and lower limbs (Figure 1, Video 1, available online at www.cmaj.ca/cgi/content/full/179/4/387/DC1). The results of laboratory investigations revealed severe hypothyroidism, which was successfully managed with thyroid hormone replacement therapy.

Severe hypothyroidism is rarely seen in clinical practice in the developed world because of the widespread availability of thyroid-stimulating hormone and assays to detect thyroid hormone. Symptoms of hypothyroidism include fatigue, cold intolerance, dyspnea, weight gain, constipation, hair loss, dry skin and menstrual irregularities. Typical findings on physical examination include dry coarse skin, periorbital and pedal edema, bradycardia, thin hair and pleural effusions.

Delayed relaxation of deep tendon reflexes (Woltman sign) ${ }^{1}$ is seen in about $75 \%$ of patients with hypothyroidism and has a positive predictive value of $92 \%$ in overtly hypothyroid patients. ${ }^{2}$ In unaffected patients, the relaxation time for deep tendon reflexes is 240-320 ms. Delays in relaxation time in patients with hypothyroidism appears to be proportional to the level of thyroid-hormone deficiency. As sensitive blood assays become more widely available around the world, the Woltman sign is likely to become obsolete as a diagnostic tool.

Clinical images are chosen because they are particularly intriguing, classic or dramatic. Submissions of clear, appropriately labelled high-resolution images must be accompanied by a figure caption and the patient's written consent for publication. A brief explanation (300 words maximum) of the educational significance of the images with minimal references is required.
Sanju Cyriac MD

Sydney C. d'Souza MD

Dhiraj Lunawat MBBS

Pai Shivananda MD

Mukundan Swaminathan MBBS

Department of Internal Medicine

Kasturba Medical College

Mangalore, India

\section{REFERENCES}

1. Houston CS. The diagnostic importance of the myxoedema reflex (Woltman's sign). CMAJ 1958;78:108-12. Available: www.pubmedcentral.nih .gov/picrender.fcgi?artid=1829539\&blob type $=$ pdf (accessed 2008 June 30).

2. Zulewski H, Müller B, Exer P, Miserez A, Staub J. Estimation of tissue hypothyroidism by a new clinical score. Evaluation of patients with various grades of hypothyroidism and controls. $J$ Clin Endocrinol Metab 1997;82:771-6. 